\title{
Diagnosis of acromegaly: room for improvement
}

Despite improvements in diagnostic tests, acromegaly remains clinically underrecognized and underdiagnosed, Reid and colleagues report. "The clinical, biochemical and tumor size characteristics at diagnosis of patients [with acromegaly] have not changed," says co-researcher Pamela Freda from Columbia University, New York, NY, USA.

Freda explains that the likelihood that therapy will be successful is related to tumor size and disease severity, so early recognition of acromegaly and initiation of treatment is essential. Improvements have been made to diagnostic tests for acromegaly, such as MRI scans that can identify small pituitary tumors, and use of sensitive biochemical markers (growth hormone and insulin-like growth factor I). Whether these advances enable clinicians to identify the disease earlier is, however, unknown.

"We conducted [a] study to determine if the clinical presentations of patients with acromegaly had changed over the last 26 years," Freda comments. Reid et al. retrospectively assessed data from 324 consecutive patients with acromegaly who were surgically treated at two New York City hospitals between 1981 and 2006. Additional data were prospectively collected from 200 of these patients during interviews and examinations conducted before and/or after treatment. Patients were divided into two groups according to the date of surgery: an early group of 108 patients (mean age 43 years) underwent surgery between January 1981 and December 1994, and a late group of 216 patients (mean age 47 years) underwent it between January 1995 and December 2006.

The researchers observed no changes in the prevalence of symptoms and signs of acromegaly at diagnosis through the study period. "Most patients still have marked manifestations of acromegaly at diagnosis, suggesting that acromegaly remains clinically under-recognized," comments Freda. Around 96\% of patients in each group presented with enlarged hands, feet and/or changes in facial features, all indicative of advanced disease; comorbidities, preoperative growth hormone levels and tumor size also remained similar in both groups.

Freda says that "increased awareness by health care professionals, especially primary care physicians, of acromegaly presentations is needed." The team plan to undertake initiatives to facilitate diagnosis of acromegaly. "With improved recognition we hope that acromegaly can be diagnosed earlier and [be treated] more successfully," Freda concludes.

\section{Lisa Richards}

Original article Reid, T. J. et al. Features at diagnosis of 324 patients with acromegaly did not change from 1981 to 2006; acromegaly remains under-recognized and under-diagnosed. Clin. Endocrinol. (Oxf). doi:10.1111/ j.1365-2265.2009.03626.x 